Bull. Chem. Soc. Ethiop. 2014, 28(2), 265-270.

Printed in Ethiopia

ISSN 1011-3924

DOI: http://dx.doi.org/10.4314/bcse.v28i2.10

(C) 2014 Chemical Society of Ethiopia

\title{
SOME QUANTUM CHEMICAL STUDY ON THE STRUCTURAL PROPERTIES OF THREE UNSYMMETRICAL SCHIFF BASE LIGANDS
}

\author{
Iran Sheikhshoaie $^{1^{*}}$, VahidSaheb $^{1}$ and Parisa Iranmanesh ${ }^{2}$ \\ ${ }^{1}$ Department of Chemistry, Shahid Bahonar University of Kerman, Iran \\ ${ }^{2}$ Department of Chemistry, Payame Nour University of Kerman, Iran
}

(Received May 30, 2013; revised November 9, 2013)

\begin{abstract}
In this studythree Schiff base ligands derived of 2-hydroxybenzaldehyde: $\mathrm{H}_{2} \mathrm{~L}^{1}$, $\mathrm{H}_{2} \mathrm{~L}^{2}$ and $\mathrm{H}_{2} \mathrm{~L}^{3}$ have been subjected to theoretical studies by using density functional theory (DFT) calculations. The electronic properties such as highest occupied molecular orbital (HOMO), lowest unoccupied molecular orbital (LUMO) energy, HOMO-LUMO gap energy, RHF energy, some of important bond lengths and angles, dipole moment and molecular densities have been investigated. Also the calculated IR spectra of these ligands are in good consistency with the experimental results.
\end{abstract}

KEY WORDS: Schiff base, 2-Hydroxybenzaldehydeand, 2-Aminoethanthiol, HOMO, LUMO, Band gap

\section{INTRODUCTION}

Schiff's base ligands have been in the chemistry catalogue for over 150 years [1]. The literature clearly shows that the study of this diverse ligand system is linked with many of the key advances made in inorganic chemistry. Not only have they played a seminal role in the development of modern coordination chemistry [2], but they can also be found at key points in the development of structural and theoretical chemistry [3-7], inorganic biochemistry [8], catalysis [9-11] and optical materials [12]. Also Schiff base ligands and some of their complexes have been extensively investigated for more than a century and have been employed in areas that include analytical and bioinorganic chemistry, non-linear optics, fluorescence studies, catalysis and materials chemistry [13-18].

The molybdenum Schiff base complexes have continued to play the role of one of the most important models of molybdoenzymes [19]. Complexes containing cis-dioxomolybdenum(VI) fragment dominate coordination chemistry of molybdenum(VI) [20]. Depending on the preparation method, molybdenum precursor and reaction conditions, the complexes synthesized with dibasic tridentate Schiff bases are of the type: monomeric - $\mathrm{Mo}(\mathrm{O})_{2}(\mathrm{~L})(\mathrm{S})$, which is dominant, where $\mathrm{L}=$ Schiff base, $\mathrm{S}=$ monodentate neutral ligand [21], or dimeric - $[\mathrm{MoO}(\mathrm{m}-$ $\mathrm{O})(\mathrm{L})]_{2}$ with asymmetric double oxygen bridge [22]. On the other hand quantum chemistry calculations have been shown to be useful in the description of the relationship among the electronic structure of the molecular systems and their optical response [23].

In this paper we report some calculated structural parameters for three salen type Schiff base ligands 2-((E)-(2-mercaptoethylimino) methyl)-4-methoxyphenol $\left(\mathrm{H}_{2} \mathrm{~L}^{1}\right)$, 2-((E)-(2-mercaptoethylimino) methyl) phenol $\left(\mathrm{H}_{2} \mathrm{~L}^{2}\right)$ and 2-((E)-(2-mercaptoethylimino)methyl)-4-nitrophenol $\left(\mathrm{H}_{2} \mathrm{~L}^{3}\right)$ (Scheme 1). These compounds have been described by Syamal [24].

${ }^{*}$ Corresponding author. E-mail: i_shoaie@yahoo.com 
Materials and reagents

\section{EXPERIMENTAL}

2-Hydroxy-5-methoxybenzaldehyde, 2-htydroxybenzaldehyde, 2-hydroxy-5-nitrobenzaldehyde, 2 -aminoethanthiol and ethanol were used as received. Methanol was distilled from magnesium methoxide. All reactions and workups were carried out under air.

\section{Instrumentation}

The IR spectra were measured as $\mathrm{KBr}$ pellets on a Perkin-Elmer 780 spectrophotometer (4000$400 \mathrm{~cm}^{-1}$ ). UV-Vis spectra were recorded as a solution (DMSO) on a 160 Shimadzu spectrophotometer. ${ }^{1} \mathrm{H}-\mathrm{NMR}$ with using $\mathrm{d}^{6}$-DMSO spectra were recorded on a Brucker Avance $500 \mathrm{MHz}$ instrument. Micro analyses for $\mathrm{C}, \mathrm{H}$, and $\mathrm{N}$ were determined on a Heracuse CHN rapid analyzer.

\section{Synthesis of Schiff base ligands}

In this research, we prepared three salen-type Schiff base ligands. 2-((E)-(2-mercaptoethylimino) methyl)-4-methoxyphenol $\left(\mathrm{H}_{2} \mathrm{~L}^{1}\right), 2-((\mathrm{E})-(2-$ mercaptoethylimino) methyl) phenol $\left(\mathrm{H}_{2} \mathrm{~L}^{2}\right)$ and 2-((E)-(2-mercaptoethylimino) methyl)-4-nitrophenol $\left(\mathrm{H}_{2} \mathrm{~L}^{3}\right)$ as literatures [24]. By condensation of 2-hydroxy-5-methoxybenzaldehyde, 2-htydroxybenzaldehyde or 2-hydroxy-5nitrobenz- aldehyde $(0.01 \mathrm{~mol}, 1.52 \mathrm{~g}, 1.22 \mathrm{~g}$ or $1.67 \mathrm{~g})$ and 2-aminoethanthiol (0.01 mol, 0.77 $\mathrm{g}$ ) in $40 \mathrm{~mL}$ methanol and the mixtures were refluxed for $4 \mathrm{~h}$ (Scheme 1). The three imines prepared were filtered and washed several times with cold methanol and dried in vacuum. Then the imines were readily purified by recrystallization from ethanol. ${ }^{1} \mathrm{H}$ NMR, UV-Vis spectroscopy and microanalyses were used to characterize these ligands. Some physical property of $\mathrm{H}_{2} \mathrm{~L}^{1}, \mathrm{H}_{2} \mathrm{~L}^{2}$ and $\mathrm{H}_{2} \mathrm{~L}^{3}$ Schiff base compounds are given in Table 1 and the relevant results of the FT-IR measurements are given in Table 2.

Table 1. Some important properties with $\mathrm{C}, \mathrm{H}$ and $\mathrm{N}$ analysis of $\mathrm{H}_{2} \mathrm{~L}^{1}, \mathrm{H}_{2} \mathrm{~L}^{2}$ and $\mathrm{H}_{2} \mathrm{~L}^{3}$ Schiff base ligands.

\begin{tabular}{|c|c|c|c|c|c|c|}
\hline $\begin{array}{c}\text { Schiff base and } \\
\text { their complexes }\end{array}$ & Formula & $\begin{array}{c}\text { Formula weight } \\
\left(\mathrm{gmol}^{-1}\right)\end{array}$ & Color & $\begin{array}{c}\text { Yield } \\
(\%)\end{array}$ & $\mathrm{C}, \mathrm{H}$ and N analyses Exp.(Theor.) & $\begin{array}{c}\text { m.p. } \\
\left({ }^{\circ} \mathrm{C}\right)\end{array}$ \\
\hline $\mathrm{H}_{2} \mathrm{~L}^{1}$ & $\mathrm{C}_{10} \mathrm{H}_{13} \mathrm{NO}_{2} \mathrm{~S}$ & 211 & Yellow & 85 & $56.2(56.87), 6.0(6.16), 6.4(6.63)$ & $118-120$ \\
\hline $\mathrm{H}_{2} \mathrm{~L}^{2}$ & $\mathrm{C}_{9} \mathrm{H}_{11} \mathrm{NOS}^{2}$ & 181 & Yellow & 91 & $59.5(59.66), 6.1(6.07), 7.7(7.73)$ & $112-115$ \\
\hline $\mathrm{H}_{2} \mathrm{~L}^{3}$ & $\mathrm{C}_{9} \mathrm{H}_{10} \mathrm{~N}_{2} \mathrm{O}_{3} \mathrm{~S}$ & 226 & Orange & 56 & $47.6(47.78), 4.4(4.42), 12.2(12.38)$ & $130-133$ \\
\hline
\end{tabular}

Table 2. Some important absorption bands in the FT-IR spectra $\left(400-4000 \mathrm{~cm}^{-1}\right)$ for $\mathrm{H}_{2} \mathrm{~L}^{1}, \mathrm{H}_{2} \mathrm{~L}^{2}$ and $\mathrm{H}_{2} \mathrm{~L}^{3}$ Schiff base ligands.

\begin{tabular}{|c|c|c|c|}
\hline Schiff base and their complexes & $v_{\mathrm{C}=\mathrm{N}}\left(\mathrm{cm}^{-1}\right)$ & $v_{\mathrm{OH}}\left(\mathrm{cm}^{-1}\right)$ & $v_{\mathrm{C}-\mathrm{H}(\mathrm{minin})}\left(\mathrm{cm}^{-1}\right)$ \\
\hline $\mathrm{H}_{2} \mathrm{~L}^{1}$ & 1638 & 3287 & $2835-2995$ \\
\hline $\mathrm{H}_{2} \mathrm{~L}^{2}$ & 1631 & 3306 & 2905 \\
\hline $\mathrm{H}^{2} \mathrm{~L}^{3}$ & 1612 & 3303 & $2976-2926$ \\
\hline
\end{tabular}

The ${ }^{1} \mathrm{H}$ NMR spectra (DMSO as an internal solvent): $\left[\mathrm{H}_{2} \mathrm{~L}^{1}: \delta(6.8,6.9\right.$, d, and 5.9, s, for aromatic hydrogen, 3, 3.9 aliphatic hydrogen, 3.8 methoxy protons and 8.4, s, for SH proton)], $\left[\mathrm{H}_{2} \mathrm{~L}^{2}: \delta(7.2,7.4, \mathrm{t}\right.$ aromatic hydrogen $5.8, \mathrm{~s}, \mathrm{H}$ imine group, 3.9, $\mathrm{t}$, aliphatic hydrogen, 7.0, $\mathrm{s}$, $\mathrm{SH}$ group)], $\mathrm{H}_{2} \mathrm{~L}^{3}: \delta(7,8.1$, d aromatic hydrogen, $2.8, \mathrm{~d}$ and 3.3 , t, aliphatic hydrogen, 8.3, $\mathrm{s}$, $\left(\mathrm{SH}\right.$ group)]. The UV/Vis (DMSO), $\lambda_{\max }, \mathrm{nm}\left(\log \varepsilon, \mathrm{L} \mathrm{mol}^{-1} \mathrm{~cm}^{-1}\right): 2 \mathrm{H}_{2} \mathrm{~L}^{1}: 486(4.81), \mathrm{H}_{2} \mathrm{~L}^{2}$ : 449(4.72), $\mathrm{H}_{2} \mathrm{~L}^{3}:$ 437(4.37). 


\section{Theoretical study}

Quantum chemical calculations (Density Functional Theory, B3LYP/6-31G (d)) were used to purposed the electronic parameters for $\mathrm{H}_{2} \mathrm{~L}^{1}, \mathrm{H}_{2} \mathrm{~L}^{2}$ and $\mathrm{H}_{2} \mathrm{~L}^{3}$ Schiff base ligands. All density functional theory (Becke's hybrid HF-DFT procedure [25] with the Lee-Yang-Parr [26] correlation functional [B3LYP/6-31G(d)]) calculations were done by using Gaussian 03 program package [27], and all three structures of these compounds were optimized (Scheme 2). Some theoretical calculated structural properties for $\mathrm{H}_{2} \mathrm{~L}^{1}, \mathrm{H}_{2} \mathrm{~L}^{2}$ and $\mathrm{H}_{2} \mathrm{~L}^{3}$ Schiff base ligands are shown in Table 3. Table 4 shows some optimized geometric parameters of $\mathrm{H}_{2} \mathrm{~L}^{1}, \mathrm{H}_{2} \mathrm{~L}^{2}$ and $\mathrm{H}_{2} \mathrm{~L}^{3}$ Schiff base ligands. Some important experimentally observed picks are given in Table 5 and compared with the most intense vibrational frequencies calculated at the B3LYP/6-31G(d) level of theory.

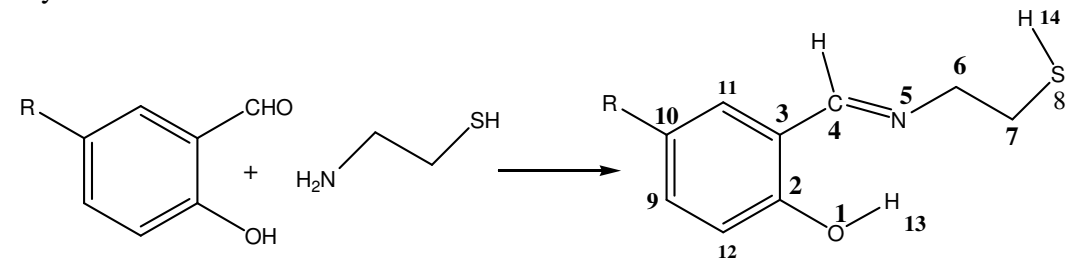

$\mathrm{R}=\mathrm{OCH} 3\left(\mathbf{H}_{2} \mathbf{L}^{1}\right), \mathrm{R}=\mathrm{H}\left(\mathbf{H}_{2} \mathbf{L}^{2}\right), \mathrm{R}=\mathrm{NO}_{2}\left(\mathbf{H}_{2} \mathbf{L}^{3}\right)$

Scheme 1. The preparation of $\mathrm{H}_{2} \mathrm{~L}^{1}, \mathrm{H}_{2} \mathrm{~L}^{2}$ and $\mathrm{H}_{2} \mathrm{~L}^{3}$ Schiff base ligands.

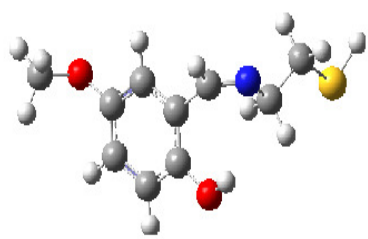

$\mathrm{H}_{2} \mathrm{~L}^{1}$

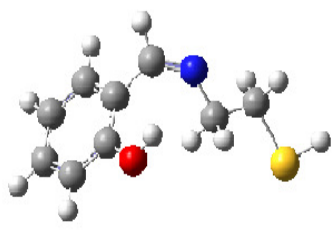

$\mathrm{H}_{2} \mathrm{~L}^{2}$

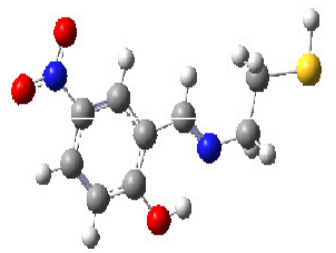

$\mathrm{H}_{2} \mathrm{~L}^{3}$

Scheme 2. The optimized structures of $\mathrm{H}_{2} \mathrm{~L}^{1}, \mathrm{H}_{2} \mathrm{~L}^{2}$ and $\mathrm{H}_{2} \mathrm{~L}^{3}$ Schiff base ligands.

Table 3. Some calculated electronic parameters for $\mathrm{H}_{2} \mathrm{~L}^{1}, \mathrm{H}_{2} \mathrm{~L}^{2}$ and $\mathrm{H}_{2} \mathrm{~L}^{3}$ Schiff base ligands.

\begin{tabular}{|c|c|c|c|c|c|c|c|c|}
\hline Compound & \multicolumn{2}{|c|}{ Mulliken atomic charges } & \multirow{2}{*}{\begin{tabular}{c} 
EHOMO \\
\cline { 2 - 7 }
\end{tabular}} & $\begin{array}{c}\mathrm{E}_{\text {LuM }} \\
(\mathrm{eV})\end{array}$ & $\mu_{\text {(Debye) }}$ & $\begin{array}{c}\mathrm{E} \\
(\mathrm{eV})\end{array}$ & $\begin{array}{c}\text { Band gap } \\
(\mathrm{eV})\end{array}$ \\
\hline $\mathrm{H}_{2} \mathrm{~L}^{1}$ & -0.6802 & -0.1679 & 0.0238 & -0.3284 & 0.0609 & 3.9019 & -26873.7000 & 0.2675 \\
\hline $\mathrm{H}_{2} \mathrm{~L}^{2}$ & -0.1961 & -0.1828 & 0.0641 & -0.3361 & 0.0608 & 2.9372 & -23776.5110 & 0.2953 \\
\hline $\mathrm{H}_{2} \mathrm{~L}^{3}$ & -0.7709 & -0.4126 & -0.0819 & -0.3573 & 0.0213 & 4.1491 & -11895.7340 & 0.3360 \\
\hline
\end{tabular}

The HOMO and LUMO orbitals are very important in defining its reactivity [28]. Higher value of HOMO of a molecule has a tendency to donate electrons to appropriate acceptor molecule with low energy, empty molecular orbitals. An electronic system with a larger HOMOLUMO gap should be less reactive than having smaller gap [29]. The larger the HOMO-LUMO orbital energy gap, the harder the molecule. The hardness has been associated with the stability of the chemical system. In the present study, the HOMO-LUMO gap the $\mathrm{HL}^{3}$ molecule is largest 
than $\mathrm{HL}^{2}$ and $\mathrm{HL}^{1}$, respectively as shown in Table 3, which clearly indicates that $\mathrm{HL}^{1}$ molecule is very reactive than the other. The calculated dipole moment values show that the $\mathrm{HL}^{3}$ molecule is highly polarin nature than the other molecules.

Table 4. Some of important optimized geometries for $\mathrm{H}_{2} \mathrm{~L}^{1}, \mathrm{H}_{2} \mathrm{~L}^{2}$ and $\mathrm{H}_{2} \mathrm{~L}^{3}$ Schiff base ligands.

\begin{tabular}{|c|c|c|c|}
\hline Bond length $(\AA)$ & $\mathrm{H}_{2} \mathrm{~L}^{1}$ & $\mathrm{H}_{2} \mathrm{~L}^{2}$ & $\mathrm{H}_{2} \mathrm{~L}^{3}$ \\
\hline $\mathrm{C} 2-\mathrm{C} 12$ & 1.388 & 1.388 & 1.394 \\
\hline $\mathrm{C} 12-\mathrm{C} 9$ & 1.381 & 1.382 & 1.375 \\
\hline C9-C10 & 1.389 & 1.393 & 1.392 \\
\hline C10-C11 & 1.379 & 1.383 & 1.381 \\
\hline C11-C3 & 1.397 & 1.397 & 1.388 \\
\hline C3-C2 & 1.390 & 1.391 & 1.407 \\
\hline C3-C4 & 1.491 & 1.491 & 1.467 \\
\hline C4-N5 & 1.265 & 1.266 & 1.266 \\
\hline N5-C6 & 1.463 & 1.463 & 1.461 \\
\hline C6-C7 & 1.520 & 1.502 & 1.525 \\
\hline C7-S8 & 1.888 & 1.888 & 1.885 \\
\hline S8-H14 & 1.354 & 1.354 & 1.354 \\
\hline C2-O1 & 1.379 & 1.377 & 1.344 \\
\hline O1-H13 & 0.950 & 0.950 & 0.966 \\
\hline H13...N5 & 2.718 & 2.706 & 1.861 \\
\hline Bond angles $\left(^{\circ}\right)$ & & & 112.981 \\
\hline H13-O1-C2 & 115.890 & 112.981 & 121.986 \\
\hline O1-C2-C3 & 123.120 & 121.986 & 122.243 \\
\hline C2-C3-C4 & 122.439 & 122.243 & 122.398 \\
\hline C3-C4-N5 & 128.287 & 122.392 & 125.128 \\
\hline C4-N5-C6 & 124.464 & 125.128 & 10.457 \\
\hline N5-C6-C7 & 107.309 & 116.457 & \\
\hline C6-C7-S8 & 108.789 & 107.688 & \\
\hline & & & \\
\hline
\end{tabular}

$\mathrm{H}_{2} \mathrm{~L}^{1}$

$\mathrm{H}_{2} \mathrm{~L}^{2}$

$\mathrm{H}_{2} \mathrm{~L}^{3}$
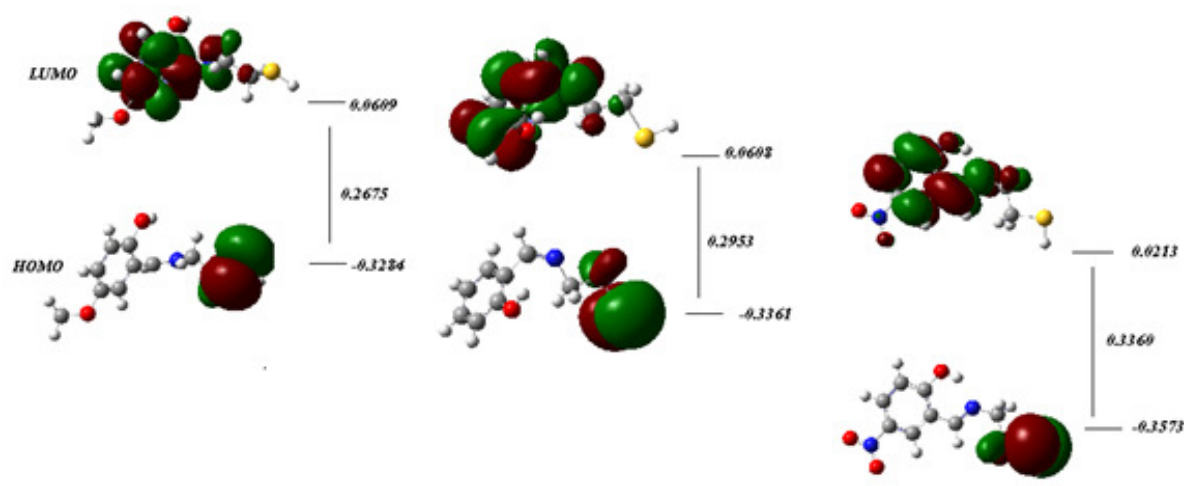

Scheme 3. Selected molecular orbital plots in various electronic excitations of $\mathrm{H}_{2} \mathrm{~L}^{1}, \mathrm{H}_{2} \mathrm{~L}^{2}$ and $\mathrm{H}_{2} \mathrm{~L}^{3}$ Schiff base ligands. 
Table 5. Some of important vibrational frequencies $\left(\mathrm{cm}^{-1}\right)$ of $\mathrm{H}_{2} \mathrm{~L}^{1}, \mathrm{H}_{2} \mathrm{~L}^{1}$ and $\mathrm{H}_{2} \mathrm{~L}^{1}$ Schiff base ligand compared with calculated.

\begin{tabular}{|c|c|c|c|c|c|c|}
\hline \multicolumn{2}{|c|}{$\mathrm{H}_{2} \mathrm{~L}^{1}$} & \multicolumn{2}{c|}{$\mathrm{H}_{2} \mathrm{~L}^{2}$} & \multicolumn{2}{c|}{$\mathrm{H}_{2} \mathrm{~L}^{3}$} & Assignment \\
\hline Experimental & Theoretical & Experimental & Theoretical & Experimental & Theoretical & \\
\hline 1180 & 1107 & 1073 & 1135 & 938 & 1030 & $\mathrm{v}_{\mathrm{C}=\mathrm{C}}$ \\
\hline 1166 & 1140 & 1047 & 1139 & 1038 & 1033 & $\mathrm{v}_{\mathrm{C}=\mathrm{C}}$ \\
\hline 3287 & 4026 & 3306 & 4025 & 3303 & 3678 & $\mathrm{v}_{\mathrm{O}-\mathrm{H}}$ \\
\hline 1638 & 1874 & 1631 & 1873 & 1612 & 1892 & $\mathrm{v}_{\mathrm{C}=\mathrm{N}}$ \\
\hline 2995 & 2983 & 2905 & 2988 & 2926 & 2953 & (aromatic) $\mathrm{V}_{\mathrm{C}-\mathrm{H}}$ \\
\hline
\end{tabular}

\section{RESULTS AND DISCUSSION}

Three Schiff base ligands $\mathrm{H}_{2} \mathrm{~L}^{1}, \mathrm{H}_{2} \mathrm{~L}^{2}$ and $\mathrm{H}_{2} \mathrm{~L}^{3}$ with NSO donor atom derived of salicylaldehyde were synthesized as literatures. A comparative study of their physico-chemical properties has been made through elemental analysis, IR, electronic spectra and ${ }^{1} \mathrm{H}$ NMR spectra. Also in the present study we have some theoretical calculations by using Gaussian 03 program to determine the best molecular configurations of the title compounds and we obtained some parameters such as the energy of the molecular orbital, $\mathrm{E}_{\mathrm{HOMO}}, \mathrm{E}_{\mathrm{LUMO}}$, dipole moment and Mulliken atomic charge. The results are given in Table 3. The calculated HOMO-LUMO orbital energies, dipole moments and total energy are very important physical parameters for chemical reactivity and biological activities of the studied compounds. The energy of HOMO is often associated with the electron-donating ability of a molecule; high values of $\mathrm{E}_{\mathrm{HOMO}}$ are likely to indicate a tendency of the molecule to donate electrons to appropriate acceptor molecules with energy and empty molecular orbital. Therefore, the energy of LUMO indicates the ability of the molecule to accept electrons. Table 3 indicates $\mathrm{O}(1), \mathrm{N}(5)$ and $\mathrm{S}(8)$ are the coordination sites for these ligands. Following the $\mathrm{E}_{\text {Номо }}$ from Table 3 and Figure 3 looks apparently easier for the molecular to offer electrons to unoccupied d-orbital of a metal ion. The higher HOMO energy from $\mathrm{H}_{2} \mathrm{~L}^{1}>\mathrm{H}_{2} \mathrm{~L}^{2}>\mathrm{H}_{2} \mathrm{~L}^{3}$ can be used as one of the parameters for the explanation of its good coordination performance, and $\mathrm{R}$ group has an important role in the complex formation by these ligands. ${ }^{1} \mathrm{H}$ NMR spectra were recorded in DMSO as an internal solvent for these ligand in our laboratory, $\left[\mathrm{H}_{2} \mathrm{~L}^{1}:(6.8,6.9, \mathrm{~d}\right.$, and 5.9, s, for aromatic hydrogen, 3, 3.9 aliphatic hydrogen, 3.8 methoxy protons and 8.4, s, for $\mathrm{SH}$ proton) $],\left[\mathrm{H}_{2} \mathrm{~L}^{2}:(7.2,7.4, \mathrm{t}\right.$ aromatic hydrogen $5.8, \mathrm{~s}, \mathrm{H}$ imine group, 3.9, t, aliphatic hydrogen, 7.0, s, SH group)], $\mathrm{H}_{2} \mathrm{~L}^{3}:(7,8.1$, d aromatic hydrogen, 2.8, $\mathrm{d}$ and 3.3, t, aliphatic hydrogen, 8.3, s,(SH group)]. Figure 3 shows the highest molecular orbitals (HOMO) for these ligand as have major contribution from the S atomic orbital of S-H group and the lowest molecular orbital (LUMO) have major contribution from the salen rings. Table 5 shows a good agreement between the experimental with theoretical data.

\section{REFERENCES}

1. Yamada, S. Coord. Chem. Rev. 1999, 192, 537.

2. Holm, R.H. J. Am. Chem. Soc. 1960, 82, 5632.

3. Sheikhshoaie, I.; Stoeckli-Evans, H.; Akbari, A.; Yasrebi, S.A.; Ebrahimipour, S.Y. Arab. J. Chem. 2012, 5, 173.

4. Sheikhshoaie, I.; Langer, V.; Yasrebi, S.A. Acta Cryst. 2011, E67, m839.

5. Hatefi, M.; Sheikhshoaie, I.; Moghadam, M.; Mirkhani,V.; Kia, R. Acta Cryst. 2010, E66, $\mathrm{m} 726$.

6. Niaz, N.; Iran, S.; Abdolrez, R. Acta Cryst. 2010, E66, m202. 
7. Sheikhshoaie, I.; Ebrahimipour, S.Y.; Sheikhshoaee, M.W. J. Sci. \& Tech. 2012, 9, 207.

8. Niederhoffer, E.C.; Timmons, J.H.; Martell, A.E. Chem. Rev. 1984, 84, 137.

9. Rezaeifard, R.; Sheikhshoaie, I.; Monadi, N.; Stoeckli-Evans, H.J. Eur. J. Inorg. Chem. 2010, 799.

10. Hatefi, M.; Moghadam, M.; Mirkhani, V.; Sheikhshoaei, I. Polyhedron 2010, 29, 2953.

11. Rezaeifard, R.; Sheikhshoaie, I.; Monadi, N.; Alipour, M. Polyhedron 29, 2010, 2703.

12. Zhang, W.; Loebach, J.L.; Wilson, S.R.; Jacobsen, E.N. J. Am. Chem. Soc. 1990, 112, 2801.

13. Sabater, M.J.; Alvaro, M.; Garcia, H.; Palomares, E.; Sherrington, J.C. Chem. Soc. Rev. 1999, $28,85$.

14. Splan, K.E.; Massari, A.M.; Morris, G.A.; Sun, S.S.; Eina, E.R.; Nguyen, S.T.; Hupp, J.T. Eur. J. Inorg. Chem. 2003, 2348.

15. Hupp, J.T. Eur. J. Inorg. Chem. 2003, 10, 2348.

16. Cozzi, P.G.; Dolci, L.S.; Garelli, A.; Montalti, M.; Prodi, L.; Zaccheroni, N. New J. Chem. 2003, 27, 692 .

17. Dey, S.K.; Mondal, N.; El Fallah, M.S.; Vicente, R.; Escuer, A.; Solans, X.; Font-Bardia, M.; Matsushita, T.; Gramlich, V.; Mitra, S. Inorg. Chem. 2004, 43, 2427.

18. Lecren, L.; Wernsdorfer, W.; Li, Y.T.; Vindigni, A.; Miyasaka, H.; Cle'rac, R. J. Am. Chem. Soc. 2007, 129, 5045.

19. (a) Holm, R.H., Kennepohl, P.; Solomon, E.L. Chem. Rev. 1996, 96, 2239;(b) Collison, D.; Garner, C.D.; Joule, J.A. Chem. Soc. Rev. 1996, 25, 25.

20. Stiefel, E.I.; Wilkinson, G.; Gillard, R.D.; McCleverty J.A. (Eds.), Comprehensive Coordination Chemistry, Vol. 3, Pergamon Press: Oxford; 1987; pp 1375-1420.

21. (a) Rajan, O.A.; Chakravorty, A. Inorg. Chem. 1981, 20, 660, (b) Topich, J.; Inorg. Chem. 1981, 20, 3704.

22. Sobczak, J.M.; Glowiak, T.; Zio, J.J. Transition Met.Chem. 1990, 15, 208.

23. Sajan, D.; Joc, H.; Jayakumar, V.S.; Zaleski, J. J. Mol. Struc. 2006, 785, 43.

24. Syamal, A. Transition Met. Chem. 1978, 3, 259.

25. (a) Koch, W.; Holthausen, M.C. A Chemist,s Guide to Density Functional Theory, 2nd ed., Wiley-VCH: Velang GmbH, Weinheim (Germany); 2000. (b) Becke, A.D. J. Chem. Phys. 1993, 98, 5648.

26. Lee, C.; Yang, W.; Parr, R.G. Phys. Rev. 1988, B37, 758.

27. Frisch, M.J.; Trucks, G.W.; Schlegel, H.B.; Scuseria, G.E.; Robb, M.A.; Cheeseman, J.R.; Montgomery, J.A.; Vreven, T.; Kudin, K.N.; Burant, J.C.; Millam, J.M.; Iyengar, S.S.; Tomasi, J.; Barone, V.; Mennucci, B.; Cossi, M.; Scalmani, G.; Rega, N.; Petersson, G.A.; Nakatsuji, H.; Hada, M.; Ehara, M.; Toyota, K.; Fukuda, R.; Hasegawa, J.; Ishida, M.; Nakajima, T.; Honda, Y.; Kitao, O.; Nakai, H.; Klene, M.; Li, X.; Knox, J.E.; Hratchian, H.P.; Cross, J.B.; Adamo, C.; Jaramillo, J.; Gomperts, R.; Stratmann, R.E.; Yazyev, O.; Austin, A.J.; Cammi, R.; Pomelli, C.; Ochterski, J.W.; Ayala, P.Y.; Morokuma, K.; Voth, G.A.; Salvador, P.; Dannenberg, J.J.; Zakrzewski, V.G.; Dapprich, S.; Daniels, A.D.; Strain, M.C.; Farkas, O.; Malick, D.K.; Rabuck, A.D.; Raghavachari, K.; Foresman, J.B.; Ortiz, J.V.; Cui, Q.; Baboul, A.G.; Clifford, S.; Cioslowski, J.; Stefanov, B.B.; Liu, G.; Liashenko, A.; Piskorz, P.; Komaromi, L.; Martin, R.L.; Fox, D.J.; Keith, T.; Al-Laham, M.A.; Peng, C.Y.; Nanayakkara, A.; Challacombe, M.; Gill, P.M.W.; Johnson, B.; Chen, W.; Wong, M.W.; Gonzalez, C.; Pople, J.A. Gaussian 03, Revision C.01, Gaussian, Inc.: Wallingford CT; 2004.

28. Fukui, K.; Yonezaw, T.; Shingu, A.; J. Chem. Phys. 1952, 20, 722.

29. Kutaran, R.; Sinem, O.; Akin, A.; Polyhedron 2007, 26, 5069. 